\title{
Discussion on Historical Data Processing Based on Real-time Database System
}

\author{
De Wu
}

Sanjiang University, Nanjing Jiangsu, 210012, China

Keywords: Real-time database system, Historical data, Storage, Treatment.

\begin{abstract}
With the continuous development of science and technology, the applied range of real-time database becomes more and more extensive, it can construct an effective bridge between traditional database technology and real-time system, ensure the optimizing operation of management information system, and the completeness of historical data can be all-round upgraded. It should be noted that in the process of preserving historical data, users need to have high frequency access to the historical data, need to combine real-time database to carry out comprehensive treatment for historical data. This paper made an analysis on the historical data connotation and store procedure, made a concentrated discussion on the data treatment mechanism based on real-time database system at the same time with historical data treatment system, aims to provide more valuable reference advice for technical researchers.
\end{abstract}

\section{Introduction}

There are only three widely used real-time database products all over the world: PI of OSI, PHD of HONEYWELL and IP21 of AspenTech in the US. Under the development background of the bid data age, applying different real-time database can solve practical issues. Especially in industrial manufacture, the data task must be managed and regulated in real time to effectively maintain the sharing mechanism and control effect of data, thus to ensure the confined structure can meet actual requirements. Though during the operation process, traditional real-time information control system also can support time limit instruction, while the targeted structure is relatively simplify, its relationship is rather stable and can realize effective forecast processing, not involve large data analysis and shared structure. For the industrial real-time database system, the most important core function of historical data processing is to construct historical record of data analysis, it is based on its high frequency use, management and integration of control application and real-time requirements can be realized. In order to decrease the reading burden of the whole system, it needs to record little recent historical data, that is to store historical data, delete data beyond limits to set up complete data analysis structure and transfer system.

On one hand, data is directly recorded in the memory unit, the application of control software and real-time optimizing software can manage the frequently accessed information on a certain extent, in virtue of memory access speed, combine real-time access demand to make selective management for historical data to ensure control system meet the actual standard. Besides, system can selectively store historical data in the historical database of the disc according to requirement, to lay a solid foundation for the easy access and treatment of data. On the other hand, using disc historical data to store disc files to effectively read its data analysis structure, for long storage time and a large amount of historical data can establish more effective treatment mechanism and control measure, ensure management model confirms to standard and also provide guarantee for the overall optimizing of the backup management project. To further optimize the operation effect of disk history database, upgrade transfer structure and backup buffer management, ensure the stability of read performance, it is necessary to conduct in-depth analysis and research on the structure and data buffer area of historical data files, focus on compression algorithm and so on. In the foreign PI, real-time database technology has a very wide development market, whether its performance or function extension direction has realized the comprehensive optimization of technology. It is worth mentioning that the PI project mainly uses the crushing algorithm and secondary filtration technique, which can 
effectively choose data structures that meet data compression technology requirements and treatment effect.

In the process of analyzing real-time database, because real-time database collect data from below by virtue of interface software, data will be simultaneously written in the main memory database and disk historical database, corresponding measurement point configuration can be confirmed effectively, also lays a solid foundation for the complete integration of stored data. After the length of stored data exceeds stipulate value, it is need to replace the old data structure, to ensure treatment effect and the completeness of data analysis system, also provide convenience for data compression. During the process of real-time database interface software processing, it needs to collect data and transfer information through OPC communication method, during information collection, data collected by interface software is different data unit for different structure and application path. During copy process, files by virtue of module upgrade, begins to practice specific principles. Before process data being stored in the disk, technicians need to make a comprehensive analysis and concentrate integration for data, improve effectiveness through compression treatment .That is to say, in the actual work development, specific compression method need to combine the completeness of data compression algorithm in the data structure operation. In order to ensure the process completeness of store in disk, it is necessary to make one time processing for historical data. Interface software simultaneously control memory-based history database and data control system, data compression module and data buffering module, providing guarantee for the all-round upgrade of historical data file[1].

\section{Historical data processing categories}

In order to make whole process analysis and control for disk historical database, during the disk history database management process, it is necessary to read historical data in different No. disk and keep it in the format of magnetic disk file, the design structure mainly includes historical data file and management information file, different file type can be applied in different fields. Real-time database project can form effective data management system by virtue of more management information file and historical data file. Besides, the storage no. of historical data file and index information are important path to set up data link frame, which needs technicians to combine real problems to make critical-path analysis and comprehensive treatment, ensure the practical treatment effect of time data file to ensure data treatment level confirms expectation.

Firstly, in order to describe disk historical database, first need to ensure the effectiveness of historical data file. Historical data file normally is the first page of the file, which recorded the index position and data pages of this data file. That is to say, by virtue of index link can index some time historical data page, by using binary search or sequential search to effectively implement positioning operation, ensure historical data lock effect. Then, in order to effectively manage information file and realize fast position historical data parameter in a certain time period, and ensure the effectiveness of information file structure, upgrade historical data file management effect, practice one-to-many relation structure.

Secondly, in order to analyze disk historical data buffer to ensure not occur frequently read disk area problem. Place historical data that will be written in disk inside the buffer, after a certain amount, write the relevant data at once. Therefore, in the system, it is necessary to combine practical situation to set up storage time. Only after system finished, can data in the historical data buffer all be written into data file structure to realize the application effect of upgrading historical data ${ }^{[2]}$. Only by analyzing the size of the buffer zone by system parameters can the user analyze and integrate the data application speed and specific project, and then implement the dynamic buffer zone ${ }^{[3]}$. Especially in the measurement configuration, set different measurement points according to requirements, thus to ensure the length of the memory history data meets the actual requirements, the allocation of data space for configuration information is carried out, data entered into the memory database are effectively compressed, the pretreatment effect conforms to the standard. After data being collected through interface software, to conduct in-depth analysis and integration, thus to be effectively added 
to the memory database, after the data area is filled in, obsolete old data, ensure new collected data can display its real effectiveness.

\section{Historical data processing based on real-time database system}

\section{Building overall structure}

In the process of real-time scheduling, it needs to operate and control through the module management system, therefore, it is necessary to establish a sound dynamic link library structure to ensure real-time database system can play its real value. In the process of establishing the overall frame structure, in order to further satisfy the real-time module control effect, need to manage the disk history data, focus on in-depth integration of management information files and historical data file. After the interface module runs, read-write operation module is used to compress the data efficiently, thus to establish data page storage mechanism, and make centralized management for information file access system and management information file, retrieve the historical data file access module, give play to the advantage of the disk history data buffer, finally read the historical data file. It should be noted that in the actual application process, interface module is not just the basic unit of data transfer and processing, it is also the basic path for the external call module to provide the interface function, relevant project managers are required to give high priority. Only to optimize the read-write operation module fundamentally, can effectively master the core of the historical library, at the same time with optimize the read-write function to conduct in-depth analysis and centralized processing for the upper interface module. It is worth mentioning that at the same time with reading point data for compressed file on the disk, also need to add data analysis mechanism and to carry out i n-depth investigation and control for the start time of the data and disk history data analysis structure ${ }^{[4]}$.

\section{Building functional modules}

In the process of comprehensive analysis of data structure, in-depth analysis and centralized integration of functional modules should be carried out. Firstly, while providing a full-range read and write operation for historical data, read and write operation function module can ensure the stability and effectiveness of data processing, to lay a solid foundation for system optimization and upgrading. And can read the relevant core data for a certain period of time through this module[5]. Secondly, compression function module, in the process of establishment and operation of industrial control mechanism, the control structure should be coordinated, measurement points can integrate a lot of real-time data, because there are too much data, in the process of space management, compression processing is a very necessary process. At present, common compression structure mainly includes loss compression and lossless compression, the former is the process of screening, sifting through a large amount of data in the disk history library with loss compression , thus to determine the data that needs to be saved to the historical data file and the data to be discarded. The latter is to encode the raw data, on the basis of saving storage space to keep the data intact. Thirdly, analyzing data page storage module can make real-time operation of the page, mainly deals with historical data pages and data index pages, provide guarantee for data access module code optimization.

\section{Conclusion}

All in all, historical data processing mechanism based on real time database system, to upgrade the management effect needs to actively implement more systematic control measures and information integration methods, optimize the use of buffer zones and memory-based history database, ensure data integrity, meanwhile, improve the sharing of data, achieve a comprehensive upgrade of the technology, and lay a solid foundation for the sustainable development of data analysis system.

\section{References}

[1] Xia Jiali. There is no conflict concurrency control protocol in embedded database system. CCCP, Computer Research And Development, 2014,41(11), pp.1936-1941. 
[2] Zhai Mingyu, Wang Jin, Wu Qingxi etc. Power distributed real-time database system architecture and key technologies, Automation of Electric Systems, 2013,37(02), pp.67-71.

[3] Jia Zhiping, Cui Wenjing. The distributed real-time database system based on the concurrent control of the Web,Journal of Dalian University of Technology, 2015,43(z1), pp.197-200.

[4] XiangJun, Li Guohui. Real-time database system service quality management based on energy consumption,Journal of Huazhong University of Science and Technology (JCR Science Edition) ,2015,36(02), pp.67-71.

[5] Yu Ge, Feng Shan. Real-time data object timing consistency service quality algorithm based on delay scheduling, Computer Application,2016,36(06), pp.1645-1649.

[6] Ding Wei. Research and Development of New DCS configuration software real-time database, Shandong University ,2015.

[7] Cao Zijian, Rong Xiaofeng. Processing methods and performance analysis of real-time database asynchronous increment data, Journal of Xi'an Technological University, 2016,32(07), pp.536-539.

[8] Liu Dagang, Wang Yongkun. Allication of Wonderware factory's real-time database on stone steel, Annual conference proceedings of national metallurgical rolling process automation technology exchange meeting and metallurgical automation information network in 2015.2015, pp.500-503.

[9] Huangmei, Tangsheng, Jiang Hongcheng etc., Technical proposal of control and integration platform and its Load analysis based on real-time database, Science and wealth, 2016,11(11), pp.76-76,77.

[10] He Yi, Wu Aiguo. Research on real-time database system structure of monitoring and control configuration software, Manufacturing Automation, 2015,29(01), pp.81-83.

[11]Han,Song,Lam et al.On Co-Scheduling of Update and Control Transactions in Real-Time Sensing and Control Systems: Algorithms, Analysis, and Performance, IEEE Transactions on Knowledge and Data Engineering, 2013, 25(10), pp.2325-2342.

[12]SUBHASH BHALLA.Parallel Concurrency Control Activity for Transaction Management in Real-time Database Systems, Journal of supercomputing, 2015,28(03), pp.345-369. 